Stefan Weber Optionen einer

\title{
konstruktivistischen Medienethik angesichts des aktuellen Trash-Fernsehens
}

Der Beitrag beschäftigt sich mit aktuellen Formaten des Trash- und Trivial-TVs in seinen unterschiedlichen Spielarten: mit Reality-TV und Doku-Soaps, mit Quiz- wie mit Gerichts-Shows. Die Konstruktion von Authentizität als verbindende Klammer all dieser Formate legt eine Betrachtung im Rahmen einer konstruktivistischen Medienethik nahe. Diese könnte sich heute mehr denn je als „Entzauberungsprogramm“ für die Programme (und die Programmierten!) der Realität des Fernsehens verstehen.

\section{Was ist heute, was ist morgen ,in'? - Zur sinkenden Halbwertszeit aktueller Fernsehformate}

Wenn es eine Konstante gibt in der Erfolgsgeschichte televisueller Formate der vergangenen fünf Jahre, dann wohl nur die, dass (mit Ausnahme vielleicht der Harald-Schmidt-Show) nichts mehr konstant ist. Oder, um es weniger paradox $z \mathfrak{u}$ formulieren: Die einzige Konstante scheint die sinkende Halbwertszeit, das immer kürzer werdende Verfallsdatum von massenwirksamen Formaten $\mathfrak{z u}$ sein. Immer neue Formate werden in Denkfabriken (d.h. von Produktionsfirmen) erfunden, an Sender verkauft, im Kontext einer multimedial-vielkanaligen Vermarktungsmaschinerie gnadenlos gehypt, ausgeschlachtet und - flugs von anderen Formaten abgelöst. ${ }^{1}$ Waren im Jahr 2000 „Big Brother" und verwandte RealLife-Überwachungsformate im Brennpunkt der Zuschauer-Aufmerksamkeit, so erleben wir im Moment eine Renaissance der Quiz-Shows und einen bis vor kurzem wohl nicht voraussehbaren Boom der GerichtsShows.

Es ist rückblickend interessant, wie schnell solche Karrieren verlaufen

1 Klaus Mertens technisches Theorem der Medienevolution („Je mehr Medien entstehen, umso schneller entstehen noch mehr Medien." [Merten 1994, 153]) müsste vielleicht aktuell um die Genre-Ebene ergänzt werden: Je mehr televisuelle Formate entstehen, umso schneller entstehen noch mehr televisuelle Formate. Ein Unterschied wäre freilich, dass sich neue technische Medien zu alten in der Regel komplementär, neue Formate $z \mathfrak{u}$ alten hingegen substitutiv verhalten. 
- und wie schnell man als Beobachter selbst in die AufmerksamkeitsFalle der Verabsolutierung stolpert. Im Jahr 1999 (wahrscheinlich einige Monate früher verfasst) erschien folgende Spekulation von Udo Göttlich und Jörg-Uwe Nieland: „Sendungsformate wie ,The Real World“ auf MTV verdeutlichen bereits jetzt, daß potentiell der Alltag von, jedermann' gefilmt und problematisiert werden kann. Eine solche Entwicklung könnte dazu fuihren, daß die mit den Soaps verbreiteten Lebensstilmuster zur Basis von Alltagsorientierungen werden." (Göttlich/Nieland 1999, 326).

Nur kurze Zeit später war „Big Brother“ in aller Munde - so, als gelte es einen Paradigmenwechsel, ja fast schon eine Achsenzeit in der Fernsehgeschichte auszurufen. Das Spektrum der Publikationen reichte von tendenziell kulturkritisch orientierten (vgl. Weber [F.] 2000) bis zu eher deskriptiv-analytischen (bisweilen auch affirmativ zu lesenden) Sammlungen (vgl. etwa Balke/Schwering/Stäheli 2000). Dass in dieser Zeit sowieso jeder Intellektuelle seinen ,Senf" zu „Big Brother" abgeben musste, versteht sich von selbst - den Autor eingeschlossen (vgl. Weber 2001a-c).

Doch die Wogen haben sich unerwartet schnell geglättet, und findige Fernsehmacher haben es gleich gewusst, dass das Endemol-Produkt eine äußerst kurze Halbwertszeit haben würde. Reality-Shows á la „Big Brother" wurden innerhalb der televisuellen Aufmerksamkeitsökonomie längst bereits von Shows neuen Typs abgelöst - etwa von Quiz-Shows ${ }^{2}$ oder Gerichts-Shows. Die Lehre aus „Big Brother“ wäre somit hier und jetzt, in jenen Shows, die derzeit en vogue sind, nicht erneut Indikatoren für einen nachhaltigen paradigmatischen Wandel zu sehen. Das hyperkommerzielle Affekt-Fernsehen, das ausschließlich auf Publikumsbindung via Emotionalität hin orientiert ist, muss offensichtlich unablässig neue Formate erfinden, die mutmaßlich immer rascher von alternativen Formaten abgelöst werden. ${ }^{3}$ Dabei kann das Pendel einmal in Richtung Quiz, dann wieder in Richtung Alltag, dann wieder in Richtung Talk ausschlagen.

In einer Meldung der deutschen Presse-Agentur vom 29. November 2001 heißt es etwa: „Von Januar 2002 an wird RTL II keine Programme

2 So wurde etwa für die ehemalige Daily-Talkerin Sonja Zietlow die Quiz-Show „Der Schwächste fliegt!“ designt, über die es allerdings im November 2001 in der österreichischen Fernsehzeitschrift „tv-media“ auch schon wieder hieß: „Bleiben Sonjas Quoten derart mies (oft nur $10 \%$ Marktanteil), wird die Rateshow nächstes Jahr eingestellt. Derweil wird bei RTL schon an einer neuen Show für Sonja Zietlow gearbeitet.“

3 Wobei das ,Neue', da das Repertoire nun einmal endlich ist, dann immer öfter das Altbekannte in neuem oder radikalisiertem Design sein wird, siehe etwa Quiz-Shows. 
ausstrahlen, ,in deren Mittelpunkt die Darstellung von Sex steht', hieß es. Vielmehr wolle sich der Sender auf das ,Echte-Leute-Fernsehen' mit Reihen wie ,Schnulleralarm', ,Popstars' oder ,Big Brother' konzentrieren." - Hört, hört! Es kann uns im Jahr 2002 also bereits eine erste echte Renaissance des Reality-Überwachungs-TVs bevorstehen!

Relativ unabhängig davon, ob der Bildschirm nun von Reality-Soaps, Quiz-Shows oder Gerichts-Shows dominiert wird, scheint es verbindende Elemente unter all diesen Formaten zu geben. Die Aufgabe einer kritischen Medienwissenschaft muss es sein, diese herauszuarbeiten.

1) Offensichtlich geht es einzig und allein um cash statt content (oder höchstens um cash via content). Diese Erkenntnis ist keineswegs trivial und kann nicht oft genug wiederholt werden. Privatwirtschaftlich organisierte Fernsehsender - und besonders die hier thematisierten Formate - haben ausschließlich eine sich selbst tragende Funktion: die kaufkräftige Zielgruppe der 14- bis 49jährigen zu adressieren, durch immer neue ,Attraktoren' wirksam zu koppeln und der werbetreibenden Wirtschaft somit ein optimales Umfeld zu bieten.

2) Verbindendes dramaturgisches Element etwa zwischen den RealityShows und den Gerichts-Shows ist die Inszenierung von Authentizität: die Befriedigung der Sehnsucht nach ungeschminktem, echtem Leben, nach realen Ereignissen und Vorkommnissen. Urteilsinstanzen, die über Recht oder Unrecht bzw. über die Rechtmäßigkeit von Handlungen entscheiden, sind dabei entweder das Publikum, das Kandidaten hinauswählen kann, oder eben die Fernsehrichter, die Urteile in nachgestellten Verhandlungen fällen. In beiden Fällen wird jedoch ein naiver Realismus suggeriert, der gerade aus konstruktivistischer und mehr noch aus non-dualistischer Perspektive problematisch ist (dazu weiter unten).

3) Aktuelle Fernsehformate gehorchen einem minutiösen Aufmerksamkeits-Design. Keine Sekunde wird hier dem Zufall überlassen, alles unterliegt einer präzise getimten Choreographie. Das folgende Zitat von SAT-1-Chefredakteur Jörg Howe bezieht sich zwar auf Fernsehnachrichten, ist aber in seiner Drastik dennoch erhellend und ohne Zweifel auch auf Unterhaltungs-Formate übertragbar: „Im Nachrichtenblock werden zwischen fünf und sieben Themen abgehandelt in einer Zeit von jeweils 20 Sekunden. Dann hängt der Bau der Sendung sehr stark davon ab, die Zuschauer zu halten. Das heißt, wir sind z.B. mit unserer Nachrichtensendung in einem sehr stark Zappingorientierten Umfeld, d.h. dem Vorabend. Hier wandern ZuschauerStröme hin und her. Die Zuschauer sind bereit, innerhalb von Sekunden den Sender, in den sie sich eingeschaltet haben, wieder $z \mathfrak{u}$ verlassen und woanders hin zu wechseln. Das heißt, es kommt darauf 
an, auch mit einer Nachrichtensendung die Zuschauer möglichst lange $z \mathfrak{u}$ binden. Wir tun das, indem wir nach dem Block etwa auf den Umschaltzeitpunkt von RTL eine wichtige, publikumsattraktive Geschichte setzen." Und präziser erklärt Howe: „Ähnlich funktioniert es zum Umschaltzeitpunkt um 18:45 Uhr, wenn die Zuschauer normalerweise zu RTL Aktuell gehen bzw. zuschalten. Auch hier versuchen wir durch den Bau der Sendung, mit einem meist konsumorientierten Thema - Beispiel neue Handy-Tarife oder ,Wird telefonieren billiger dadurch, dass die Post das Recht auf die letzte Meile verliert' - Zuschauer zu halten und zu binden, und zwar über den Umschaltzeitpunkt 18:45 Uhr hinaus, so dass wir einen nächsten logischen Bruch in der Sendung um 18:47 und 30 Sek. anbieten, wenn wir an den Sport übergeben. Wir merken dann deutlich, dass sich Zuschauerpotential und Zuschauerverhalten verändern."4

4) Aktuelle Fernsehformate sind in den meisten Fällen in einen multimedialen und vielkanaligen Kontext eingebettet: Netz- und Printmedien, CDs und Merchandising-Artikel jedweder Art bilden zusammen genommen ein Netzwerk der Cross-Promotion (vgl. dazu jüngst auch Böhme-Dürr/Sudholt 2001), das sich ebenfalls selbstreferenziell trägt. Innermediales Agenda-Setting wird zum immer bedeutenderen Faktor: Der neue Richter in der neuen SAT-1 Gerichts-Show tritt als Gast in der "Harald-Schmidt-Show" auf, wo natürlich vorher auch schon alle anderen Talker und Quizmaster Gäste waren (zur Selbstreferenzialität bei Harald Schmidt siehe Frieske 1998).

Aus medienwissenschaftlicher Sicht sind somit (ökonomisch plausible) Selbstreferenzialität und Konstruktivität (als Inszenierung von Authentizität) die entscheidenden Faktoren erfolgreicher Trash-Produkte: Selbstreferenzialität meint den (steigenden) Bezug von Medien auf Medien, Konstruktivität das (zunehmende) bewusste Designen von medialen Wirklichkeiten. Beide Trends sind nicht nur für die Macher ökonomisch, sondern auch für das Publikum Garanten für leichte Unterhaltung.

4 Vortrag von Jörg Howe, Chefredakteur von Sat.1, zum Thema "Was und wer hat Fernseh-Nachrichtenwert?" auf der Tagung „Das ist uns wichtig?! Wer und was die Medienöffentlichkeit beschäftigt" der Akademie der Diözese Rottenburg-Stuttgart, 18. und 19. Oktober 2001 (Zitat aus dem Vortragsmanuskript, S. 10f.; persönliche Übermittlung). 


\section{Trash-TV und die Rolle der Medienwissenschaft}

Medienwissenschaftliche Anschlussdebatten können zumeist zwei binären Fronten zugeordnet werden: Entweder, es wird kulturpessimistisch-moralisierend argumentiert; oder aber es wird eine affirmativ-positive Sichtweise gewählt. Erstere Kommentatoren müssen sich in der Regel den Vorwurf gefallen lassen, einfach nicht auf der Höhe der Zeit zu sein oder die integrativen Funktionen des Trash-Fernsehens zu verkennen. So schrieb etwa der österreichische Kolumnist Hans Rauscher in einer Qualitätszeitung, dass man in der Analyse des Erfolgs von "Taxi Orange“ (dem österreichischen „Big Brother"-Klon, siehe jüngst auch Flicker 2001) "mit jeder kulturpessimistischen Kritik am falschen Dampfer ist“ („Der Standard“, 2. November 2000, S. 1), denn: „Hier lernt der junge Mensch durch teilnehmende Beobachtung gruppendynamisches Verhalten." Und über die empirische Studie „Das Phänomen ,Big Brother'“ von Jürgen Grimm urteilt Peter Moritz in den „Medienimpulsen“: „Die Studie liest sich wie ein Werbeprospekt für ,Big Brother', nach dessen Lektüre man sich eigentlich nichts sehnlicher wünschen müsste, als dass das Fernsehen derartige Produkte verstärkt ausstrahlt [...] ". (Moritz 2001, 19). Dies ist also die andere Seite der Medaille: Medienwissenschaftliche empirische Forschung, die - in mittlerweile längst ebenfalls ideologischer reflexartiger Abkehr von der Ideologie der Kulturkritik - sich einzig und allein auf die positiven Aspekte der Aneignung von Trash-TV konzentriert. Begriffe und Konzepte wie „Manipulation“, „Verführung"5 oder auch nur "Medienmacht" haben hier keine Chance.

Gibt es einen dritten Weg? Hat die Medienwissenschaft auf die gegenwärtigen televisuellen Formate auch mit einer kritischen Betrachtung reagiert, die nicht gleich in dumpfen Kulturpessimismus zurückfällt bzw. diesem Vorwurf zumindest zu entgehen vermag? - Es scheint nur die binäre Alternative zu geben: Entweder, man argumentiert aus (neo-)marxistischer, politökonomischer oder kulturkritischer Sicht Adorno'scher Provenienz, oder aber man bewegt sich - in Überwindung derselben - im emanzipatorischen Sprachspiel der Cultural Studies. Eine dritte Option, die Fruchtbarmachung von Konstruktivismus und Systemtheorie, wurde indes mit wenigen Ausnahmen (Rademacher 2001 und Weber 2001d) kaum verfolgt.

Dazu kommt ein zunehmend rein deskriptives Verständnis von Wissenschaft, das die Diskussion über präskriptive Aspekte, über Werte und Normen von vornherein auszuschließen scheint. Darf ein Medienwissenschaftler ein Urteil darüber abgeben, was ein ,gutes' und was ein ,schlechtes' Medienprodukt ist?6 Kann ein Wissenschaftler Qualitätsurteile fällen, darf er einen Sollen-Satz formulieren? Oder muss er 
dann immer erklären, dies nicht in der Rolle des Wissenschaftlers, sondern als Privatperson zu tun?

\section{Auf dem Weg zu einer konstruktivistischen Medienethik ...}

Ich möchte die präskriptive Dimension forcieren und dies im Kontext einer konstruktivistischen Medienethik begründen. Unter Konstruktivismus verstehe ich, wie in verschiedenen Zusammenhängen dargelegt (etwa Weber [S.] 2000, 42ff.), vor allem eine empirische Theorie, die von empirisch zu beobachtender steigender Konstruktivität einer bestimmten Beobachtungsinstanz ausgeht. Es geht also um keine All-Aussage im Sinne von „Unsere Welt ist eine Erfindung" oder „Die Realität ist immer schon eine Konstruktion". Solche auf erkenntnistheoretischer Ebene geführten Debatten zwischen Realisten und Konstruktivisten sind mühsam und münden zwangsläufig in Pattstellungen. ${ }^{7}$ Es ist ein bedeutender Unterschied, ob man - auf (erkenntnis-)theoretischer Ebene - sagt, die Realität sei immer schon, an sich oder per se eine Konstruktion, oder aber ob man davon ausgeht, dass die Realität zunehmend, immer mehr konstruiert werde. Wenn der Konstruktivismus Letzteres behauptet, geht er dann nicht von einem realistischen Verständnis von Realität aus, die auch konstruktionsfrei existierend oder gar erkennbar wäre? Um dieses Problem zu umgehen, habe ich vorgeschlagen, auf erkenntnistheoretischer Ebene den Konstruktivismus durch den Non-Dualismus (zur Grundlegung vgl. Mitterer 1992) zu ersetzen. Dieser Ansatz stellt epistemologisch nicht mehr die Frage, ob die Realität abgebildet oder konstruiert werde, er interessiert sich vielmehr auf der Meta-Ebene für das $\mathrm{Zu}$ -

5 Zu ergänzen wären klarer Weise: Verdummung, Verblödung, Verflachung u.v.a. verwandte Trendbeobachtungen.

6 Diese Frage stellt sich auch Prokop (2001) - allerdings erneut aus einer viel zu negativen und simplifizierenden Perspektive. Überdies vertritt er einen besonders naiven Realismus, wenn er etwa schreibt: „Wir müssen sehen, wie die Welt heute wirklich funktioniert. Wir müssen sehen, wie die Welt der Medien wirklich ist." (Prokop 2000, 10)

7 Josef Mitterer hat dies schön auf den Punkt gebracht: Der Realist behauptet in der Regel: „Der Konstruktivist ist in Wirklichkeit ein Realist - und spätestens dann, wenn er mit dem Kopf durch die Wand will, müßte er dies auch eingestehen." (Mitterer 1998, 554) Der Konstruktivist hingegen kann immer kontern: „Wenn die Welt zu 100\% die Welt meiner Erfahrung und mein Erleben ist, wenn Wissen ausschließlich eine interne Konstruktion des menschlichen Subjekts ist, dann gilt dies auch für den Realisten, ob er dies wahr haben will oder nicht: Der Realist ist in Wirklichkeit ein Konstruktivist." (Ebd.) 
standekommen von Unterscheidungen wie Realität und Konstruktion oder Abbild und Konstrukt.

Wird Konstruktivismus also als eine empirisch zu beweisende Theorie von zunehmender Konstruktivität (in einem gegebenen Zeitraum, von einer gegebenen Beobachtungsinstanz) begriffen, dann scheint es, dass eine konstruktivistische Ethik die Aufgabe hätte, als ,reale Realitäten' präsentierte konstruierte Wirklichkeiten methodisch zu ,entzaubern'. Konstruktivistische Ethik interessiert sich also für die Konstruktionsund Inszenierungsregeln von Wirklichkeit und Authentizität, sie ,outet ${ }^{\text {‘ }}$ Design, wo Echtheit ,verkauft' wird. - Von einem anderen, eben erkenntnistheoretischen oder neurobiologischen konstruktivistischen Verständnis herkommend wurde unter konstruktivistischer Ethik bislang meist eine Ethik der Sicherung und Maximierung von Pluralität verstanden (vgl. etwa Hejl 1995, 41). Heinz von Foersters ethischer Imperativ heißt dann auch konsequenterweise: „Handle stets so, daß die Anzahl der Möglichkeiten wächst" (zitiert nach Hungerige/Sabbouh 1995, 143).

Nun scheint jedoch unser aktuelles Problem gerade das des Erstickens in Multioptionalität zu sein. Haben wir nicht (zumindest im massenmedialen Kontext) schon genug Handlungsoptionen - können wir nicht bereits zwischen mehr als 30 TV-Kanälen, zahllosen Großkinos, endlosen Chats und Foren usw. wählen? Ist nicht gerade die vielbeschworene postmoderne Unübersichtlichkeit, Kontingenz und Vielfalt das Problem, das zu neuen Selektionsmodi zwingt? Soll konstruktivistische Ethik also dieser Pluralität und Konstruktivität huldigen, oder geht es nicht vielmehr darum, diese zu problematisieren?

Das Paradoxe ist, dass wir gerade in dieser fragmentarisierten Verschiedenheit wieder einen kleinsten gemeinsamen Nenner vorfinden, wir also erneut alle gleich sind: Wenn alle postmodern different sind, ist genau das die verbindende Identität. Dies ist der ambivalente Effekt von "Big Brother“. Gerhard Johann Lischka hat dies so beschrieben: „Wird heute in den Medien jede/r zum potentiellen Star (in der Reality Show etc.), giert die Masse nach dem (fast) extraterrestrischen Status, so wird aus einer so genannten Demokratie eine Ochlokratie, in der das Prinzip der Gleichheit zum Gefangensein in der Masse (genau genommen der Gruppe $=$ ochlos), zur Zementierung, zur Erstarrung jeglicher Bewegung verkommt." (Lischka 2001, 19)

Im Konstruktivismus Siegfried J. Schmidts ist dieser (scheinbare paradoxe) Egalisierungs-Effekt qua postmoderne Pluralisierung mit dem Begriff des „Programms“ gut umschrieben: Ein Kulturprogramm ist in Schmidts Konzeption ein Set von Verhaltensanweisungen (vgl. etwa Schmidt 2001, 186), das primär implizit, subtil funktioniert denn sichtbar wirksam wird. Lassen sich aus dieser theoretischen Perspektive 
Phänomene wie die (immer zeitlich begrenzt zu denkende) Big-BrotherHysterie erklären?

Aus konstruktivistischer Sicht erscheint es somit wichtig, den Authentizitäts-Anspruch von Trivial-TV zu dekonstruieren: Forschungen, die sich mit dem Inszenierungscharakter der Shows, mit der Überlagerung von Realität durch Theatralität, von Natürlichkeit durch Künstlichkeit usw. beschäftigen, erscheinen zentral und gefordert. Präskriptive Sätze und Werturteile sollten dabei keineswegs unter ein ,Denkverbot' fallen.

\section{... und zu einer Renaissance der Medienkritik?}

Vieles von dem, was in Shows wie "Big Brother" oder „Der Schwächste fliegt!" gezeigt wurde und wird, verweist auf medienethisch höchst problematische Zusammenhänge: eine lustvolle, fast schon perverse Unterwerfung unter Überwachung (Stichwort Überwachungs- und Disziplinargesellschaft), Mobbing als vermeintlicher Erfolgs- und Aufstiegsgarant (Stichwort Ellbogengesellschaft), Hang zu Personenkult und Autoritarismus u.a. - Die Medienethik und -kritik steht dabei vor dem paradoxen Grundproblem, dass ihre Kritik scheinbar vom Mediensystem selbst absorbiert und instrumentalisiert wurde und wird: There is no bad advertising. Wie Gerald Jörns unlängst in der Telepolis schrieb: „Medienethiker forcierten die Effekte der Aufmerksamkeit noch zusätzlich, indem sie die Sendung vor dem eigentlichen Start heftig kritisierten." (Jörns 2001, 4) Gibt es einen Ausweg aus dieser Funktionalisierung von Kritik im Interesse der Fernsehmacher? Ist es Luhmannsche Autopoiesis, dass Kritik offenbar auf teuflische Weise in die Reproduktion von Trash-TV mit eingebaut wird? Sollte man deshalb als Kritiker lieber ganz verstummen?

In einer Zeit totaler Medialisierung, des Turbo-Kapitalismus und des hyperkommerziellen Affekt-Fernsehens scheint es schwer, eine Nische für einen Gegendiskurs, für ein Jenseits der Massenmedien zu finden. Wer hätte sich gedacht, dass Visionen vom „Bürgerfernsehen“ oder von "partizipativen Medien“, die auch der Autor noch zu Beginn der Neunziger mit Projekten alternativer künstlerischer Fernsehstationen mitverfolgt hat, in jenem Statisten-Spiel enden, wie es uns tagtäglich mit den Daily Talks oder eben mit Reality-TV vorgeführt wird? Sollte man resignieren, Trash-TV sich selbst überlassen und Utopien endgültig begraben?

Couragierte Medienkritik scheint ein Widerspruch in sich zu sein, weil sie (Massen-)Medien benötigt, um gehört zu werden: schon kann der Vorwurf der Hassliebe zum Objekt der Kritik erhoben werden. Wenn die 
Medienkritik diesen Einwand formallogisch entkräften könnte, wäre dies eine gute Voraussetzung für ihre Renaissance. Die Sterne dafür stehen leider nicht allzu gut.

\section{Literatur}

Balke, Friedrich/Schwering, Gregor/Stäheli, Urs (Hg.) (2000): Big Brother. Beobachtungen. Bielefeld: transcript.

Böhme-Dürr, Karin/Sudholt, Thomas (Hg) (2001): Hundert Tage Aufmerksamkeit. Das Zusammenspiel von Medien, Menschen und Märkten bei „Big Brother“. Konstanz: UVK.

Flicker, Eva (Hg.) (2001): Wissenschaft fährt Taxi Orange. Befunde zur österreichischen Reality-TV-Show. Wien: Promedia.

Frieske, Michael (1998): Selbstreferentielles Entertainment. Televisio-

näre Selbstbezüglichkeit in der Fernsehunterhaltung. Wiesbaden:

Deutscher Universitäts-Verlag.

Göttlich, Udo/Nieland, Jörg-Uwe (1999): Daily Soaps als Kaleidoskop der Individualisierung. In: Latzer, Michael u.a. (Hg.): Die Zukunft der Kommunikation. Phänomene und Trends in der Informationsgesellschaft. Innsbruck/Wien: StudienVerlag, S. 313-328.

Hejl, Peter M. (1995): Ethik, Konstruktivismus und gesellschaftliche Selbstregelung. In: Rusch, Gebhard/Schmidt, Siegfried J. (Hg.): Konstruktivismus und Ethik. DELFIN 1995. Frankfurt am Main: Suhrkamp, S. 28-121.

Hungerige, Heiko/Sabbouh, Kariem (1995): Let's talk about ethics.

Ethik und Moral im konstruktivistischen Diskurs. In: Rusch, Gebhard/Schmidt, Siegfried J. (Hg.): Konstruktivismus und Ethik. DELFIN 1995. Frankfurt am Main: Suhrkamp, S. 123-173.

Jörns, Gerald (2001): Hundert Tage Aufmerksamkeit. Wissenschaft-

liche Betrachtungen zur Reality-Show Big Brother. In: Telepolis, http://www.heise.de/tp/deutsch/inhalt/buch/11060/1.html, 23. November 2001.

Lischka, Gerhard Johann (2001): Wie kommen wir uns nahe? Zwischen Ochlokratie und Dismorphophobie. In: Lischka, Gerhard Johann/ Feuerstein, Thomas (Hg.): Media-made. Wie kommen wir uns nahe? Köln: Wienand, S. 17-26.

Merten, Klaus (1994): Evolution der Kommunikation. In: Merten, Klaus/Schmidt, Siegfried J./Weischenberg, Siegfried (Hg.): Die Wirklichkeit der Medien. Eine Einführung in die Kommunikationswissenschaft. Opladen: Westdeutscher Verlag, S. 141-162.

Mitterer, Josef (1992): Das Jenseits der Philosophie. Wider das dualistische Erkenntnisprinzip. Wien: Passagen Verlag.

Mitterer, Josef (1998): Der Radikale Konstruktivismus: „What diffe- 
rence does it make?". In: Ethik und Sozialwissenschaften, Heft 4/1998, S. 554-556.

Moritz, Peter (2001): „Big Brother“ - Verblödet uns das Fernsehen? Langeweile als Faszination. In: Medienimpulse - Beiträge zur Medienpädagogik, Heft 35, Themenheft „Reality-TV - Fragen an die Medienpädagogik“, März 2001, S. 17-23.

Prokop, Dieter (2000): Der Medien-Kapitalismus. Das Lexikon der neuen kritischen Medienforschung. Hamburg: VSA-Verlag.

Rademacher, Lars (2001): „Zeig mir, wer Du wirklich bist ..." Was wir sehen, wenn wir Big Brother sehen - Sieben Lesarten eines Medienereignisses. In: Communicatio Socialis, Heft 1/2001, S. 30-51.

Schmidt, Siegfried J. (2001): Kultur und Kontingenz: Lehren des Beobachters. In: Müller, Albert/Müller, Karl H./Stadler, Friedrich (Hg.): Konstruktivismus und Kognitionswissenschaft. Kulturelle Wurzeln und Ergebnisse. Heinz von Foerster gewidmet. Wien/New York: Springer, S. 183-192.

Weber, Frank (Hg.) (2000): Big Brother. Inszenierte Banalität zur Prime Time. Münster: Lit.

Weber, Stefan (2000): Was steuert Journalismus? Ein System zwischen Selbstreferenz und Fremdsteuerung. Konstanz: UVK Medien.

Weber, Stefan (2001a): Vom Fremdwort „Medienkritik“. Warum (nicht nur) die Kommunikationswissenschaft immer affirmativer wird. In: Telepolis, http://www.heise.de/tp/deutsch/inhalt/co/4715/1.html, 18. Januar 2001.

Weber, Stefan (2001b): Bloß keine Kritik! Die Kommunikationswissenschaft und Reality-TV. In: Neue Zürcher Zeitung, 16. Februar 2001, Medien und Informatik, S. 71.

Weber, Stefan (2001c): Kollektive Angst vor Kritik. „Taxi Orange“ \& Co: Warum ist die Medienkritik so zahnlos? In: Falter, Nr. 7/01, 16.-22. Februar 2001, S. 19-20.

Weber, Stefan (2001d): Falsch verbunden? Warum Unterhaltung immer ,realistischer' und Journalismus immer ,fiktionaler' wird. In: Medienimpulse - Beiträge zur Medienpädagogik, Heft 35, Themenheft „Reality-TV - Fragen an die Medienpädagogik“, März 2001, S. 11-16. 